ISSN: 2386-3919 - e-ISSN: 2386-3927

DOI: https://doi.org/10.14201/et20203816985

\title{
MODELOS DE INTELIGENCIA Y ALTAS CAPACIDADES: UNA REVISIÓN DESCRIPTIVA Y COMPARATIVA
}

\author{
Intelligence Models and High Capacities: \\ A Descriptive and Comparative Review
}

\author{
Alberto Quílez RoBres y Raquel LOZANO Blasco
}

Recibido: 30/06/2020; Aceptado: 26/08/2020; Publicado: 30/12/2020

Ref. Bibl. ALBERTO QUílEZ ROBRES y RAQUEL LOZANO BLASCO. Modelos de inteligencia y altas capacidades: una revisión descriptiva y comparativa. Enseñanza E Teaching, 38, 1-2020, 69-85.

RESUMEN: La relación entre la inteligencia y el rendimiento académico ha dado paso a diferentes teorías y modelos durante los últimos cuarenta años. Entre las elaboradas durante el siglo Xx destacan la Teoría de los Tres Anillos de Renzulli (1978), basada en la sobredotación; la de las Inteligencias Múltiples de Gardner (1983), que propone el desarrollo de diferentes habilidades; la Teoría Triárquica de Sternberg (1985), que presenta un carácter cognitivo, y el modelo Sea Star de Tannenbaum (1986), con un punto de vista sociocultural. Ya en el siglo XxI aparece el megamodelo de Desarrollo del Talento de Subotnik, Olszewski-Kubilius y Worrell (2012) y el modelo Tripartito de la Alta Capacidad de Pfeiffer (2015). Con el desarrollo de estos modelos, al concepto de inteligencia se añaden los de talento, superdotación, habilidades y capacidades. Este artículo realiza una revisión y comparación de los modelos más representativos vinculados a las altas capacidades por considerar que son imprescindibles a la hora de elaborar propuestas didácticas para el desarrollo de un aula de altas capacidades. Todos ellos destacan por la búsqueda de la atención a la diversidad y a la inclusión educativa de los más capaces, y también por la elaboración de propuestas que favorezcan su pleno desarrollo. teorías.

Palabras clave: capacidad cognitiva; alto rendimiento; inteligencia; modelos; 
SUMMARY: The relationship between intelligence and academic performance has given way to different theories and models for the past forty years. Among those developed during the twentieth century, Renzulli's Three Rings Theory (1978) based on giftedness, Gardner's (Multiple Intelligence) (1983) that proposes the development of different abilities, Sternberg's Triarchic Theory (1985) stand out that presents a cognitive character, and Tannenbaum's (1986) Sea Star model with a socio-cultural point of view. Already in the 21st century, the Subotnik Talent Development mega-model, Olszewski-Kubilius \& Worrell (2012) and Pfeiffer's Tripartite High Capacity model (2015) appear. With the development of these models, the concept of intelligence is added to that of talent, giftedness, abilities and capacities. This article reviews and compares the most representative models related to high capacities, considering that they are essential when preparing didactic proposals for the development of a high-capacity classroom. All of them stand out for the search for attention to diversity and the educational inclusion of the most capable, and also for the elaboration of proposals that favor their full development.

Key words: cognitive ability; high performance; intelligence; models; theories.

\section{INTRODUCCIÓN}

Las investigaciones relacionadas con el constructo de inteligencia son muy numerosas y coincidentes sobre su valor predictivo respecto al desempeño académico (Deary, Strand, Smith y Fernández, 2007; Kaufman, Reynolds, Liu, Kaufman y McGrew, 2012). Este término se ha redefinido numerosas veces a lo largo del tiempo sin que los expertos hayan llegado a un consenso. Galton (1892, en Triglia, Regader y García-Allen, 2018) fue el primero en introducirlo dentro del mundo de la psicología. Lo definió como una aptitud de carácter general y superior que permite explicar un conjunto de aptitudes especiales determinadas principalmente de manera genética. Posteriormente, Piaget (Piaget e Inhelder, 1971), en su teoría constructivista del desarrollo de la inteligencia, lo definió como un conjunto de operaciones lógicas inherentes al ser humano que le permiten percibir, clasificar, comprender, establecer inferencias, etc. No obstante, no fue hasta finales del siglo xx cuando se desarrollaron diferentes modelos estructurados de la inteligencia, la superdotación y su vinculación con el desempeño académico e inclusión educativa.

Autores como Renzulli (1978), Gardner (1983, 1985), Sternberg (1985), Tannenbaum (1986), Subotnik, Olszewski-Kubilius y Worrell (2012) y Pfeiffer (2015) presentaron diferentes teorías englobadas en tres grandes grupos: cognitivos, socioculturales y de capacitación. A su vez, se establecieron distinciones entre habilidades, talentos y capacidades (Subotnik et al., 2012), llegando a la conclusión de que el concepto de inteligencia no podía ser entendido como un elemento único e inamovible (Barraca y Artola, 2004; Gardner, 1985, Gottlieb, Hyde, ImmordinoYang y Kaufman, 2016). Vinculado al concepto de inteligencia se encuentra el término altas capacidades, definido como el rendimiento superior, en un dominio 
específico, en comparación con otros sujetos de funcionamiento de alto nivel en dicho dominio (Subotnik, Olszewski-Kubilius y Worrell, 2011).

Desde los años noventa hay abierto un debate sobre cómo son identificados y cómo deben ser atendidos los alumnos de altas capacidades (Martínez, Castejón y Sánchez, 1997). En este sentido, Sánchez, Cobeñas, Del Valle Chauvet y Belinchón (2008) consideran que hay una serie de características que los identifican: capacidad intelectual general, aptitud académica, creatividad y aptitud social. Siguiendo las directrices del Consejo Europeo de Lisboa (2000) y las conclusiones del Consejo (2009) sobre el Marco Estratégico para la cooperación europea en el ámbito de la educación y la formación (ET 2020) (Diario Oficial de la Unión Europea, 2009), la nueva escuela inclusiva tiene como objetivo último el pleno desarrollo de sus estudiantes, ofreciendo igualdad de oportunidades para el aprendizaje, incluyendo a los "más capaces" y potenciando su talento, crecimiento social y emocional. Para ello, es necesaria una pronta identificación de estos alumnos para evitar la desmotivación y el fracaso escolar. Este aspecto implica la colaboración de toda la comunidad educativa: familia, docentes, equipo de orientación, etc. (Comisión Europea, 2010; Diario Oficial de la Unión Europea, 2009).

2. Modelos ESTRUCTURAdOS DE LA INTELIGENCIA, SUPERDOTACiÓN Y DESARROLLO DE CAPACIDADES

\subsection{Teoría de los Tres Anillos de Renzulli (1978)}

Renzulli (1978) introduce un modelo de inteligencia y superdotación encaminado al rendimiento académico. Sin embargo, señala que una alta capacitación no es suficiente para asegurar un desempeño académico excepcional. En sus formulaciones teóricas aseguraba que la inteligencia debe ir acompañada de otros factores tales como la motivación y la creatividad. La unión de tres factores: inteligencia superior a la media (aptitudes), motivación (compromiso con la tarea) y creatividad forman la representación tridimensional de la superdotación simbolizada por tres anillos entrecruzados. Para Renzulli (2012), aquellos niños que desarrollan una interacción entre los tres factores requieren oportunidades y servicios especiales por parte de las instituciones educativas. Este modelo lo completará más tarde introduciendo factores de personalidad, de valores o ambientales. Por lo tanto, la definición de superdotación de Renzulli se clasificaría como multidimensional al incluir factores no intelectivos para valorar las capacidades desde una perspectiva educativa (Reyero y Tourón, 2000). Con un enfoque claramente inclusivo y basado en la atención a la diversidad, se proponía un modelo de enriquecimiento que respaldara educativamente a los alumnos superdotados. Esta teoría fue reformulada por el propio autor puntualizando algunos aspectos del primer anillo, dedicado a la capacidad por encima de la media, al considerar el elemento intelectual como un constructo puramente psicológico (Renzulli, 2012). 
Respecto a la creatividad como elemento implicado en el segundo anillo, Renzulli (1981) la define como un elemento multifactorial que incluye la originalidad de pensamiento, la capacidad de otorgar enfoques novedosos, la capacidad de inhibir los convencionalismos y los procedimientos establecidos. Sin embargo, este autor también advierte de lo complicado de su medición, y por lo tanto, no es partidario de la aplicación de test de creatividad, sino que se recurra a procedimientos de revisión de procesos pasados y presentes, comprobando la evolución creativa de la persona y estableciendo relaciones y comparaciones (Renzulli, 2012).

En cuanto al tercer anillo, presenta la implicación en la tarea como un elemento motivacional, incluyendo elementos intrínsecos y psicosociales como la persistencia, la confianza en sí mismo, la independencia y la propia motivación intrínseca (Mönks y van Boxtel, 1988; Renzulli, 2012; van Boxtel y Mönks, 1992). Con este modelo teórico convergerán posteriormente las teorías de Sternberg (1985) y la de Tannenbaum (1986) al destacar el elemento intrínseco de la persona. Se puede concluir definiendo el modelo de los Tres Anillos sobre la superdotación, como un modelo de características estáticas evidenciado mediante la referencia continua de "rasgos» (Mönks y van Boxtel, 1988; van Boxtel y Mönks, 1992).

\subsection{Teoría de las Inteligencias Múltiples. Gardner (1983)}

Gardner revolucionó el estudio de la inteligencia al realizar una crítica al concepto tradicional y elaborar la Teoría de las Inteligencias Múltiples en 1983. Tras sus estudios sobre desarrollo cognitivo y lesiones cerebrales postuló que la perspectiva planteada hasta ahora sobre el intelecto no era plausible y formuló la teoría de que los individuos tienen diferentes fortalezas y debilidades. Se introduce un mayor grado de complejidad a la hora de definir la superdotación, ya que ahora la inteligencia ya no es unitaria, sino que está compuesta por distintas capacidades específicas, distintas e independientes en función de los campos de la actividad humana (Ramos, 2008). Gardner (2000) aboga por un amplio concepto de inteligencia y la plantea como un conjunto de componentes relativamente independientes que interaccionan entre ellos. Es decir, denominará inteligencias a cada uno de "los potenciales cognitivos del individuo, en un complejo proceso de interacción entre factores biológicos y culturales» (Pérez, 2018: 360).

No existe una única inteligencia, sino varias y estas se manifiestan en diferentes culturas y entornos siendo necesario un proceso de adaptación. Por eso, cada inteligencia resultará útil en un contexto determinado, de tal manera que establece la existencia de ocho inteligencias atendiendo a diferentes regiones cerebrales implicadas, a las habilidades de carácter cultural e histórico, a la evolución, a la existencia de individuos con talentos o prodigiosos, etc. (Mora y Martín, 2007). Pero la novedad también se halla en los criterios que utiliza para identificar cada una de las inteligencias, mostrando un interés especial en el desarrollo de las mismas mediante programas educativos que se adecuen a las necesidades de cada una de ellas (Reyero y Tourón, 2000). 
ALBERTO QUÍLEZ ROBRES Y RAQUEL LOZANO BLASCO

\subsection{Teoría Triárquica de la Inteligencia. Sternberg (1985)}

Sternberg desarrolló la Teoría Triárquica de la Inteligencia en 1985 planteando una subdivisión de la misma en la que trata desde los procesos mentales utilizados hasta la capacidad de adaptación del individuo al contexto. Esta subdivisión teórica contempla la inteligencia y su desarrollo desde tres puntos de vista: a) competencial-analítica, b) experiencial-creativa y c) contextual-práctica. La inteligencia competencial la relaciona con la académica; la experiencial con la creatividad; y la contextual con la relación con el medio (Villamizar y Donoso, 2013). Esta fragmentación teórica obedece a una atención multidisciplinar de los diferentes contextos personales, atendiendo al mundo externo del individuo, al mundo interno y a la interacción o contextualización de ambos (Sternberg et al., 2010). Además de formular su teoría el autor dejó claro que la vida exitosa no dependía únicamente de la inteligencia y que este éxito podría deberse a diferentes elementos tanto intrínsecos como extrínsecos de la persona.

La subteoría competencial-analítica define el concepto de componente como un proceso básico de información que funciona sobre las representaciones internas de los objetos o símbolos (Sternberg, 1985). De tal forma que un determinado componente presentará una cierta extensión en el tiempo (duración), una mayor o menor posibilidad de correcta o incorrecta ejecución (dificultad) y una determinada probabilidad de poder ser llevado a cabo (Sternberg, 1985; Sternberg et al., 2010). También se señala la existencia de metacomponentes que son definidos como los procesos de tipo superior que se emplean para realizar una tarea planificando, controlando y tomando decisiones (Sternberg, 1985).

En el caso de la subteoría experiencial, Sternberg (1985) pone en valor la experiencia individual y las características de la tarea. El autor entiende como experiencia la tarea a realizar mediante una selección de actividades, ejercicios, etc., partiendo de lo teórico y no de lo práctico como es habitual en teorías de carácter cognitivo y diferencial. La resolución de problemas dependerá de la experiencia previa del individuo. Por lo tanto, la inteligencia no puede ser comprendida fuera de un contexto sociocultural ya que es fundamental para que el individuo se adapte al contexto real, estando sujeto a cambios culturales. Junto a la adaptación, Sternberg pone en relieve dos conceptos más: la selección y la configuración del medio. De tal manera que, si es imposible la adaptación, el individuo puede elegir, seleccionar otro medio. Si, a su vez, esto no es posible, puede tratar de reconfigurar el contexto real al que se enfrenta. El comportamiento inteligente se manifiesta en la capacidad para adaptarse a la exigencias y desafíos nuevos del entorno (Mora y Martín, 2007).

Se puede concluir que la Teoría Triárquica ofrece una definición de la inteligencia como un conjunto de procesos cognitivos inteligentes tanto en función del tiempo como del contexto y situados dentro de un marco triárquico donde se relacionan aspectos contextuales, experienciales y de componentes implicados (Martín, 1992; Sternberg et al., 2010). 
En trabajos posteriores Sternberg (1999) desarrolla su Teoría de la Inteligencia Exitosa como expansión a su teoría anterior. Presenta tres tipos de comportamiento inteligente: la inteligencia analítica (ámbito académico), la inteligencia creativa (problemas novedosos) y la inteligencia práctica (problemas de la vida cotidiana) (Pérez y Medrano, 2013). Además, Sternberg (en Sternberg y Davidson, 1986) desarrolla un modelo cognitivo al que denomina Teoría Pentagonal Implícita. Este tipo de modelo trata de identificar los procesos y estrategias que los individuos de altas capacidades utilizan para resolver tareas y que los diferencia de los demás. Establece una serie de criterios o condiciones que permita identificarlos: criterio de productividad, de rareza, de demostrabilidad, de valor y de excelencia (Del Canto, 2014; Ramos, 2008).

\subsection{Modelo Sea Star de Tannenbaum (1986)}

La inteligencia desde un punto de vista sociocultural, y, por extensión, la superdotación, no puede ser explicada sin atender al contexto social, económico, familiar, cultural, etc., en el que el individuo vive, interactúa y se desarrolla (Pérez, 2018). Este enfoque en el estudio de la inteligencia está representado por Tannenbaum (1986) y su modelo sociocultural de la inteligencia y la superdotación "Sea Star». Realizó una ampliación del concepto de alta capacidad al relacionarlo con un satisfactorio estado social, físico, moral, emocional o intelectual del individuo. El contexto social, cultural y familiar dificultan o potencian el desarrollo de las capacidades. Además, señalaba que el verdadero talento se demuestra en la edad adulta.

Tal como indica Blumen (2015), esta teoría propone cinco factores para determinar el talento y la superdotación: 1) Capacidad general: factor de inteligencia general que engloba todas las capacidades cognitivas. 2) Aptitudes específicas: múltiples factores de inteligencia que afectan a diferentes tareas. 3) Factores no intelectuales: como el autoconcepto o la motivación, relacionados con el desarrollo de la persona. 4) Influjos ambientales escolares y familiares: ambiente estimulante. 5) Factor suerte: fortuna o suerte en momentos cruciales de la vida. Estos cinco factores son un requisito necesario para conseguir un alto rendimiento, ya que cada uno por separado no es suficiente.

La visión de Tannenbaum (2003) sobre el talento es comprensiva, integradora y dinámica. Este autor opina que los niños con altas capacidades debían estar expuestos a estímulos, experiencias e informaciones variadas, ricas y abundantes durante la niñez y adolescencia con el fin de desarrollar todas sus potencialidades y talentos. Para ello, propuso diferentes medidas de atención entre las que incluía el enriquecimiento curricular.

Por otro lado, el modelo Sea Star presenta rasgos no solo de carácter cognitivo, sino también motivacionales como el compromiso, la autoconfianza, el bienestar mental, la motivación o el autoconcepto (Blumen, 2015). Al igual que la teoría de Sternberg (1985), esta tiene en consideración tanto las variables internas como las 
ALBERTO QUÍLEZ ROBRES Y RAQUEL LOZANO BLASCO

externas. Según Tannenbaum (2003), para que se diese la superdotación el desarrollo de estos dos tipos de variable debían estar presentes de manera equilibrada y gradual. De tal manera que un número elevado de unos rasgos no compensen la ausencia de otros. No obstante, hay un elemento a destacar dentro de esta teoría, el factor suerte. Para este autor existen diferentes acciones o situaciones que un individuo puede o no puede experimentar y que, a su vez, potencian o frenan un desarrollo talentoso. Por lo tanto, amplió el concepto de inteligencia, pero sobre todo estableció pautas para la correcta identificación de la inteligencia y de los individuos que presentan altas capacidades (Tannenbaum, 1986).

\subsection{Mega-Modelo de Desarrollo del Talento de Subotnik, Olszewski-Kubilius y Worrell (2012)}

Subotnik et al. desarrollaron en 2012 un modelo estructurado que, a diferencia del resto de teorías, no trataba de manera explícita la inteligencia. En este caso, los autores proponen un desarrollo de talentos, dicho de otra forma, el camino desde la potencialidad al rendimiento y a la eminencia; de la capacidad a la competencia, a la pericia y a la eminencia como proceso de desarrollo. Ahora bien, nadie garantiza la transición de la pericia a la eminencia ya que existe un porcentaje muy pequeño de éxito. Subotnik et al. (2011) definen la alta capacidad como la materialización del rendimiento extremo y superior de un dominio, de un talento específico. Destacan la importancia del desarrollo de los talentos desde las primeras etapas de la vida. En ese sentido, Sosniak (1985) señala la necesidad de convertir el potencial en competencia desde la más temprana edad. Pero Subotnik et al. (2011) van más allá y afirman que el potencial es clave en las primeras etapas educativas, para pasar después a serlo el rendimiento entendido como alta capacidad y así poder llegar a la eminencia cuando el talento está totalmente desarrollado. A lo largo de las diferentes etapas durante las que tiene lugar el proceso de desarrollo del talento el papel de padres, maestros y mentores es muy importante, destacando el de los padres en la etapa de transformación del potencial a competencia.

Para Olszewski-Kubilius, Subotnik y Worrell (2015a, 2015b) las variables cognitivas y psicosociales cobran gran importancia a lo largo de todo el desarrollo y deben ser trabajadas continuamente. Dentro de las variables psicosociales distinguen entre las limitadoras y las potenciadoras. Dentro de estas últimas destacan la motivación, las oportunidades y la mentalidad productiva. Para estos autores tan importante es la motivación como la capacidad a la hora de conseguir rendimientos de excelencia. Además, preparar para la eminencia es a lo que debe aspirar la educación, y al mismo tiempo promover las oportunidades que mediante el esfuerzo han de ser aprovechadas por los individuos de altas capacidades.

Por lo tanto, para estos autores el camino de la capacidad a la pericia e incluso el ciclo completo es un proceso de desarrollo y crecimiento personal. Además, desde un punto de vista inclusivo y de atención a la diversidad se debe considerar los diferentes niveles de partida y de velocidad del desarrollo. El talento se ha 
demostrado que no siempre se potencia de la misma manera, ni aparece en las mismas edades, obedeciendo a un criterio de singularidad y particularidad del ser humano. Hay que destacar la gran influencia que las habilidades psicosociales ejercen sobre este proceso como motoras e impulsoras del mismo. De este modo, para llevar a cabo un proceso creciente y estable en el desarrollo del talento, son elementos clave los padres, los mentores, los profesores, la regulación emocional, la retroalimentación y la enseñanza directa (Olszewski-Kubilius et al., 2015b).

\subsection{Modelo Tripartito de la Alta Capacidad de Pfeiffer (2013, 2015)}

El Modelo Tripartito de Pfeiffer (2013) es una síntesis de los modelos de desarrollo, psicométricos y de transformación. Ofrece una visión de la alta capacidad desde tres puntos de vista diferentes: «La alta capacidad vista como alta inteligencia, la alta capacidad vista como rendimiento sobresaliente y la alta capacidad vista como alto potencial para sobresalir o rendir de modo excelente" (Pfeiffer, 2015: p. 66). En un primer enfoque se puede utilizar el cociente intelectual como medida de capacidad general, pero no debe ser entendido como elemento determinante. Para Pfeiffer (2013), los niños con alta capacidad muestran una probabilidad mayor de alcanzar logros y éxitos que sus iguales en edad, experiencia y oportunidades. Del mismo modo, añadía que el alumnado con alta dotación demuestra un rendimiento superior o presenta de forma evidente un potencial significativo para sobresalir en el rendimiento académico. En ese sentido, este tipo de alumnado debería beneficiarse de programas especiales de educación (Pfeiffer, 2013, 2015).

Las tres perspectivas propuestas por Pfeiffer (2015), alto cociente intelectual, alto rendimiento y alto potencial, las fundamenta de la siguiente manera. El alto cociente intelectual será medido mediante pruebas psicométricas, pero estas puntuaciones deben ser tomadas con cautela ya que este constructo resulta artificioso a la hora de medir la superdotación que es una construcción social e irreal. Por otro lado, el alto rendimiento no estaría en total desacuerdo con la medida del cociente intelectual ya que contemplaría una capacitación general o potencialización de diferentes talentos. Además, este punto pone en relieve la excelencia académica de un alumno en su práctica diaria dentro del aula, pero tomando también como referencia la creatividad, la motivación, la persistencia, etc. En definitiva, valores intrínsecos no cognitivos en la línea de modelos anteriormente presentados y, por lo tanto, se hace necesario que para los alumnos de rendimiento sobresaliente se programen planes de estudio enriquecidos y con un reto académico elevado (McClain y Pfeiffer, 2012).

La tercera perspectiva del modelo de Pfeiffer presenta como elemento clave el potencial para sobresalir. En este punto hay que señalar que las pruebas psicométricas no son suficientes y se debe recurrir a la observación en el aula, a recabar información de familiares, profesores y del propio alumno para conocer aspectos relevantes del contexto en el que el alumno se desarrolla. Esto obedece a que no todos los alumnos superdotados han podido disponer de las situaciones positivas 
necesarias para desarrollar sus potencialidades, incluso en ocasiones se han podido ver mermadas. También porque es necesario la comprensión de que cada alumno parte de un nivel y unas circunstancias personales y únicas (Pfeiffer 2013, 2015). «La educación de los más capaces, basada en la perspectiva del potencial para la excelencia, consiste en un plan de estudios muy motivador y enriquecido que pueda incluir en realidad intervenciones compensatorias»(Pfeiffer, 2015: 85).

Este autor recuerda que su experiencia le hace conocedor de una realidad: el talento se desarrolla y se transforma con el tiempo. Además, señala la importancia de la capacidad física, del trabajo duro, la voluntad, la persistencia, la buena orientación, etc. Para Pfeiffer (2015), la alta capacidad es un constructo dinámico donde no todos los talentosos se convierten en élite porque para ello es necesario algo más que capacidad general. Por lo tanto, esta teoría ofrece tres maneras de observar la alta capacidad y recuerda que hay muchos factores que intervienen en la adquisición de la competencia y la pericia.

\section{MÉTODO}

Mediante la selección de las teorías y modelos relacionados con las altas capacidades, sobredotación y talentos de los últimos cuarenta años y con un diseño cualitativo comparativo se realizó una revisión y análisis descriptivo de los seis modelos relacionados.

\section{Resultados}

Estas teorías o modelos referidos a la inteligencia y las altas capacidades presentan diferencias y semejanzas. En la Tabla 1 se puede observar que, en cuanto a la clasificación de los modelos, tanto la teoría de Sternberg (1985) como la de Gardner (1983) son cognitivas; de capacitación o rendimiento académico son las teorías de Renzulli (1978) y Pfiffer (2013, 2015); en el modelo sociocultural se encuentra la teoría de Tannenbaum (1986), y de carácter mixto de capacitación y sociocultural la teoría de Subotnik et al. (2012). En cuanto al matiz del concepto referido a la inteligencia optan por capacidades Renzulli (1978) y Sternberg (1985). El de talento es el empleado por Tannenbaum (1986) y Subotnik et al. (2012). Como excepción, Gardner (1983) utiliza el de habilidades y, por último, Pfiffer $(2013,2015)$ indistintamente emplea talento o capacidades.

De manera general estas teorías se caracterizan por ser dinámicas, integradoras e inclusivas, y por poner su interés en el contexto en que se desenvuelven los individuos. La excepción se encuentra en la de Renzulli (1978) por su carácter estático y por no ser objeto de estudio el contexto. Creatividad y motivación aparecen como los factores valorados de forma unánime por todos los modelos revisados. Se puede resumir que, junto a la importancia de las capacidades innatas de los sujetos, las variables personales y el contexto sociocultural explican en gran medida las 
altas capacidades. En algunos casos se introducen factores como el de esfuerzo que, en definitiva, se refiere al rendimiento (Subotnik et al., 2012) y en otros casos el de suerte (Tannenbaum, 1986).

TABLA 1

Descripción y características de los Modelos de Inteligencia y Altas Capacidades

\begin{tabular}{|c|c|c|c|c|c|}
\hline AUTORES & TEORÍAS & MODELOS & CONCEPTO & CARACTERÍSTICAS & PROPUESTA \\
\hline Renzulli, 1978 & $\begin{array}{l}\text { Teoría de los } \\
\text { Tres Anillos }\end{array}$ & $\begin{array}{l}\text { Capacitación } \\
\text { o rendimiento } \\
\text { académico }\end{array}$ & Capacidades & $\begin{array}{l}\text { Multifactorial, variables } \\
\text { intrínsecas como la } \\
\text { motivación, creatividad, } \\
\text { rasgos, enriquecimiento, } \\
\text { inclusiva, estática }\end{array}$ & $\begin{array}{l}\text { Atención a la diversidad, } \\
\text { programas educativos } \\
\text { de enriquecimiento }\end{array}$ \\
\hline Gardner, 1983 & $\begin{array}{l}\text { Teoría de las } \\
\text { Inteligencias } \\
\text { Múltiples }\end{array}$ & Cognitivo & Habilidades & $\begin{array}{l}8 \text { tipos de inteligencia, } \\
\text { potencialidades, } \\
\text { contexto-cultura }\end{array}$ & $\begin{array}{c}\text { Programas educativos } \\
\text { adecuados a cada una } \\
\text { de las inteligencias }\end{array}$ \\
\hline $\begin{array}{c}\text { Sternberg, } \\
1985\end{array}$ & $\begin{array}{l}\text { Teoría Triárquica } \\
\text { de la Inteligencia }\end{array}$ & Cognitivo & Capacidades & $\begin{array}{l}\text { Contexto sociocultural } \\
\text { (adaptación), competencias } \\
\text { (rendimiento), experiencia } \\
\text { (creatividad, motivación, } \\
\text { novedad, automatización), } \\
\text { componencial, dinámica }\end{array}$ & $\begin{array}{l}\text { Identificación de estrategias } \\
\text { y procesos de resolución } \\
\text { de problemas que los } \\
\text { diferencian de los demás }\end{array}$ \\
\hline $\begin{array}{l}\text { Tannenbaum, } \\
1986\end{array}$ & Modelo Sea Star & Sociocultural & Talento & $\begin{array}{l}\text { Contexto social-cultural- } \\
\text { económico, rasgos, variables } \\
\text { internas (motivación, } \\
\text { autoconcepto), factor suerte, } \\
\text { integradora, dinámica }\end{array}$ & $\begin{array}{l}\text { Identificación, medidas } \\
\text { de atención (mentoría), } \\
\text { enriquecimiento curricular }\end{array}$ \\
\hline $\begin{array}{l}\text { Subotnik, } \\
\text { Olszewski- } \\
\text { Kubilius \& } \\
\text { Worrell, } 2012\end{array}$ & $\begin{array}{l}\text { Mega-Modelo } \\
\text { de desarrollo } \\
\text { del Talento }\end{array}$ & $\begin{array}{c}\text { Mixto: } \\
\text { Capacitación/ } \\
\text { Sociocultural }\end{array}$ & Talento & $\begin{array}{l}\text { Proceso de desarrollo } \\
\text { y crecimiento personal: } \\
\text { potencialidad, pericia, } \\
\text { eminencia, variables } \\
\text { psicosociales (motivación, } \\
\text { oportunidades, etc.), esfuerzo, } \\
\text { inclusiva, dinámica }\end{array}$ & $\begin{array}{l}\text { La educación debe aspirar } \\
\text { a la excelencia, mentoría }\end{array}$ \\
\hline $\begin{array}{l}\text { Pfeiffer, } \\
\text { 2013-2015 }\end{array}$ & $\begin{array}{l}\text { Modelo Tripartito } \\
\text { de Alta Capacidad }\end{array}$ & $\begin{array}{l}\text { Capacitación } \\
\text { o rendimiento } \\
\text { académico }\end{array}$ & $\begin{array}{l}\text { Capacidades/ } \\
\text { talento }\end{array}$ & $\begin{array}{l}\text { Valores intrínsecos } \\
\text { (motivación, creatividad), } \\
\text { información del contexto, } \\
\text { dinámica (se desarrolla y } \\
\text { transforma con el tiempo) }\end{array}$ & $\begin{array}{l}\text { Programas especiales de } \\
\text { educación acelerados, } \\
\text { enriquecidos, } \\
\text { planteamiento de retos, } \\
\text { intervenciones compensatorias }\end{array}$ \\
\hline
\end{tabular}

\section{DISCUSIÓN}

Tal como se ha podido apreciar, a lo largo de la revisión de las distintas teorías y modelos que tratan el tema de las altas capacidades, la inteligencia no debe ser entendida como un elemento único, indivisible, estático y fiablemente medible. Para Gotlieb et al. (2016) la inteligencia medida mediante pruebas de cociente intelectual no captura de manera adecuada las capacidades de los estudiantes talentosos. 
De las diversas definiciones y reformulaciones de este concepto que se han consultado para este estudio, para Sternberg (1985) es una actividad mental que va dirigida intencionadamente hacia la adaptación o la transformación del entorno en el que se vive. Este autor realiza una crítica al enfoque psicométrico y plantea otro mucho más cognitivo introduciendo la adaptación al contexto real del individuo.

Para Gardner (1983), la inteligencia consiste en la habilidad que se posee para resolver problemas que son importantes dentro de una cultura determinada. Esta teoría propone un enfoque plural y alejado del concepto tradicional de cociente intelectual, promoviendo la importancia de las diferentes capacidades y sus potencialidades y coincide con Sternberg en las consecuencias de la contextualización.

En el caso de Tannenbaum (1986), el término inteligencia se enmarca dentro de la superdotación y establece que la inteligencia no puede ser entendida sin contemplar el contexto social del individuo. Además, señala los cinco aspectos que deben concurrir para hablar de alta capacidad. En esta misma línea se encuentra la teoría desarrollada por Renzulli (1978). Para este autor, la excepcionalidad de la inteligencia se halla en el comportamiento inteligente del individuo atendiendo a tres aspectos: habilidad, compromiso con la tarea y creatividad.

Por otro lado, Subotnik et al. (2012) en una evolución del estudio intelectual olvidan de manera explícita el término inteligencia para acercarse al concepto de talento como conjunto de condiciones que componen la primera. De esta manera y enfocado a la alta capacitación dichos autores distinguen distintos niveles de desarrollo, distintos puntos de partida, distintos talentos y potencialidades y determinan diferentes caminos paralelos. El potencial apoyado en el rendimiento lleva a la eminencia; con capacidad, competencia y pericia se alcanza la eminencia, pero en todo momento contemplando el factor creativo en el desarrollo personal, procesual y productivo.

Finalmente, Pfeiffer (2015) considera una medida útil el cociente intelectual para identificar a los alumnos más dotados. No obstante, reconoce la importancia de las capacidades, actitudes, destrezas y rasgos de la personalidad como componentes esenciales de la inteligencia. Para este autor alta inteligencia, logros sobresalientes y potencial hacia la excelencia son los pilares básicos de una alta capacidad que pueden o no confluir a la vez. Por lo tanto, todas estas investigaciones suponen un avance teórico-conceptual del término inteligencia y de su vinculación a la atención educativa de los más capaces. Además, señalan la importancia de otros elementos no cognitivos como el factor social y contextual de la persona como elemento diferenciador (Pérez, 2018; Sternberg, 1999; Sternberg, Kaufman y Grigorenko, 2008).

La fundamentación teórica de la inteligencia y las altas capacidades, tal como se observa en esta revisión, recoge varios modelos que se clasifican en: cognitivos, de capacitación o rendimiento académico y sociocultural. Las diferentes teorías estudiadas se enmarcan en uno u otro modelo, pero entendiendo el aspecto intelectual como un concepto multicomponente. Para Renzulli (1978), la inteligencia y la sobredotación tienen como finalidad el rendimiento académico. Propone tres 
factores para conseguirlo: alta capacidad, creatividad y motivación (los tres anillos). Posteriormente los amplia e incluye aquellos referidos a la personalidad, a los valores y al ambiente o contexto (familia, maestros, amigos, etc.). En el caso de Sternberg (1985), divide su teoría sobre la inteligencia en tres subteorías que daban forma triárquica a dicho concepto: a) competencial-analítica, b) experiencial-creativa y c) contextual-práctica. Este planteamiento se fundamenta en la adaptabilidad otorgando gran importancia al contexto social. En esa línea se encuentra la Teoría de Tannenbaum «Sea Star» (1986), que presenta la inteligencia en su vertiente de superdotación como un constructo sociocultural que debe atender a cinco aspectos básicos (capacidad general, aspectos específicos, variables personales, contexto social y suerte).

El planteamiento teórico que ha presentado mayor controversia ha sido la teoría de Gardner (1985). Este autor considera la existencia de ocho inteligencias diferentes. La fundamenta en la posibilidad de distintas formas de ser «inteligente» atendiendo tanto a capacidades como habilidades y talentos. El estudio de Castejón, Pérez y Gilar (2010) determinó que estas «inteligencias» no son totalmente independientes de g tal y como sugería Gardner (1995), pero tampoco son unitarias como defendían sus detractores.

El siglo XXI ofrece dos enfoques que avanzan en el concepto de inteligencia. Tratan elementos que se presuponen integrados en ella como el talento y la capacidad, pero sin abordar directamente dicho constructo. Subotnik et al. (2012) y Pfeiffer (2015) propusieron sendos modelos multifactoriales dedicados a la manifestación, construcción y desarrollo de las diferentes capacidades, habilidades y talentos de la persona en la faceta de alta capacitación mediante un estudio abierto, plural, evolutivo y psicosocial. Para Subotnik et al. (2012), se trata de un desarrollo y crecimiento personal que se obtiene mediante la capacidad, la motivación y el esfuerzo. Por otro lado, Pfeiffer (2015) opina que, además de alta inteligencia, motivación y oportunidades es necesario desarrollar el potencial para obtener la excelencia.

Precisamente el aspecto social de la inteligencia, junto con la valoración, que no medición exacta, de la creatividad, reflejan un consenso dentro de los modelos revisados. La totalidad de los autores contempla la importancia del contexto sociocultural y afectivo-emocional, junto con la historia individual de la persona, como elementos potencializadores, estabilizadores o inhibidores del surgimiento exitoso. Los mismos Sternberg et al. (2010) plantean que el éxito en la vida depende de diversos factores o habilidades; que no todos son de carácter cognitivo y que, por lo tanto, no pueden ser examinados ni medidos por los test convencionales de inteligencia.

Durante más de un siglo los investigadores educativos han intentado medir y explicar la inteligencia y, por consiguiente, las aptitudes que identifican el rendimiento académico y, por extensión, el talento que lleva al éxito y la excelencia. Las teorías revisadas presentan un concepto de altas capacidades que contempla diversos aspectos tanto cognitivos como aquellos que configuran la realidad del individuo (Sahuquillo et al., 2016). La mayor parte de ellas consideran que factores como la creatividad, la motivación y la implicación en la tarea interaccionan con 
la inteligencia. Además, subrayan la importancia del contexto sociocultural para el desarrollo de las capacidades.

En definitiva, se refleja que la sobredotación es un constructo multidimensional, existiendo distintas clases de superdotación. Por otro lado, se deduce que las capacidades cognitivas son esenciales pero otras variables motivacionales y socioculturales van más allá de la inteligencia (Barraca y Artola, 2004). En resumen, se consigue expandir los límites conceptuales de la inteligencia destacando la importancia del contexto y la experiencia y se ponen en valor las variables personales motivacionales.

Los distintos autores revisados, y siempre dentro del ámbito educativo, consideran que hay que dar respuestas a estos alumnos capaces y talentosos. Estas respuestas deben estar destinadas a ofrecer oportunidades en el marco de sus potencialidades e intereses. Presentan diferentes propuestas contemplando a estos alumnos diferentes al resto y a la vez diferentes entre sí (Reyero y Tourón, 2000). Programas de enriquecimiento curricular, programas especiales acelerados, intervenciones compensatorias, programas educativos especiales adaptados a cada uno de los talentos, programas de atención personalizada (mentoría), planteamiento de retos son algunas de las propuestas. Con ellas se buscar elaborar currículos escolares diferenciados que se adapten a cada talento específico y que ayuden a desarrollarlo. Una educación de calidad que promocione la excelencia debe ofrecer oportunidades adecuadas que permitan desarrollar a estos alumnos sus potencialidades al máximo en todos los ámbitos de la vida (Reyero y Tourón, 2000). Por lo tanto, una programación didáctica dirigida a un aula de altas capacidades deberá elaborar un programa educativo adecuado a las necesidades de estos alumnos (Gardner, 2000), presentar nuevos retos y desafíos apropiados al contexto cultural donde se desarrolla (Sternberg et al., 2010), que motive al alumnado (Renzulli, 2012) mediante la exposición a estimulos buscando el equilibrio entre variables internas y externas (Tannenbaum, 2003), para generar oportunidades y preparar para la eminencia (Subotnik et al., 2011) utilizando todo tipo de recursos y una buena orientación educativa (Pfeiffer, 2015).

\section{CONClusiones}

La atención a la diversidad de los alumnos con necesidades educativas especiales, incluidos los de altas capacidades, ha supuesto un gran cambio metodológico y conceptual en la educación actual. Se ha dotado a los centros escolares de herramientas, recursos y proyectos necesarios para dicha atención obedeciendo a la directiva europea de inclusión educativa.

El objetivo de esta revisión era disponer de una información completa de las distintas teorías y modelos que tratan la inteligencia y las altas capacidad. Su finalidad era la de aplicar estos conocimientos en la elaboración de una programación didáctica de aula para alumnos de altas capacidades, en la etapa de educación primaria y secundaria, desde el departamento de Orientación y con la colaboración de los tutores de aula. 
Los autores de los diferentes modelos intelectivos presentados comparten de forma unánime la imposibilidad de medir objetivamente la inteligencia individual en sus diferentes facetas. Por lo tanto, para llegar a una aproximación de la misma, con la finalidad de detectar a los posibles talentos o superdotados, se deben utilizar métodos cuantitativos y cualitativos. Esta medición deben incluir la observación y la entrevista que recoja las opiniones de las partes implicadas de la comunidad educativa. Además, se debe buscar evidencias de los diferentes aspectos competenciales, que no solo serán de carácter factorial o cognitivo, sino también de carácter contextual social y personal. Al revisar sus propuestas se observa cómo se desvinculan claramente de las teorías tradicionales de la inteligencia y justifican la existencia de distintos factores que dan paso a nuevos conceptos como son alta capacidad, sobredotación o talentos. En la actualidad el concepto de inteligencia se entiende como un elemento plural, diversificado, con una estructura factorial multicomponente donde convergen operaciones superiores de carácter cognitivo en convivencia con el contexto sociocultural y afectivo-emocional del individuo. Además, contempla el desarrollo de capacidades y talentos teniendo como objetivo el logro académico, un desarrollo pleno y el bienestar vital, siempre con un carácter integrador que incluya a los más capaces.

Por lo tanto, una programación didáctica dirigida a un aula de altas capacidades deberá elaborar un programa educativo adecuado a las necesidades de estos alumnos (Gardner, 2000), presentar nuevos retos y desafíos apropiados al contexto cultural donde se desarrolla (Sternberg et al., 2010), que motive al alumnado (Renzulli, 2012) mediante la exposición a estímulos buscando el equilibrio entre variables personales de carácter interno o externo (Tannenbaum, 2003), para generar oportunidades y preparar para la eminencia (Subotnik et al., 2011) utilizando todo tipo de recursos y una buena orientación educativa (Pfeiffer, 2015). Por otro lado, la mayoría de los autores sugieren que para conseguir el desarrollo pleno del talento o altas capacidades es necesario comenzar en edad temprana a propiciar las condiciones necesarias para el mismo. De ahí la importancia de implantar planes y programas de desarrollo de altas capacidades en las aulas de Educación Primaria y Secundaria.

\section{REFERENCIAS BIBLIOGRÁFICAS}

Barraca, J. y Artola, T. (2004). La identificación de alumnos con altas capacidades a través de la EDAC. [The identification of students with high capacities through the EDAC]. eduPsykhé, 3(1), 3-18. http://repositorio.ucjc.edu/bitstream/handle/20.500.12020/100/ C00028507.pdf?sequence $=1$.

Blumen, S. (2015). Abraham Tannenbaum: 1924-2014. Revista de Psicología (PUCP), 33(1), 231234. http://www.scielo.org.pe/scielo.php?pid=S0254-92472015000100009\&script=sci_ arttext\&tlng=pt.

Castejón, J. L., Pérez, A. M. y Gilar, R. (2010). Confirmatory factor análisis of Project Spectrum activities. A second-order g factor or multiple intelligences? Intelligence, 38(5), 481-496. https://doi.org/10.1016/j.intell.2010.07.002. 
ALBERTO QUÍLEZ ROBRES Y RAQUEL LOZANO BLASCO

MODELOS DE INTELIGENCIA Y ALTAS CAPACIDADES: UNA REVISIÓN DESCRIPTIVA Y COMPARATIVA

Comisión Europea. (2010). Europa 2020: Una estrategia para un crecimiento inteligente, sostenible e integrador. [A strategy for smart, sustainable and inclusive growth]. Comunicación 3.3.2010-2020. Bruselas, Bélgica. https://eur-lex.europa.eu/legal-content/ES/ TXT/?uri=LEGISSUM\%3Aem0028.

Deary, I. J.; Strand, S.; Smith, P. y Fernandez, C. (2007). Intelligence and educational achievement. Intelligence, 35, 13-21. https://doi.org/10.1016/j.intell.20006.02.001.

Del Canto, M. C. G. (2014). Perfil de la motivación hacia el aprendizaje en alumnado con alta capacidad. [Profile of motivation towards learning in students with high capacity]. International Journal of Developmental and Educational Psychology, 6(1), 277-287. https://www.redalyc.org/pdf/3498/349851790032.pdf

Diario Oficial de la Unión Europea. (2009). Conclusiones del Consejo del 12 de mayo de 2009 sobre un marco estratégico para la cooperación europea en el ámbito de la educación y la formación (ET 2020), [Council conclusions of 12 May 2009 on a strategic framework for European cooperation in the field of education and training (ET 2020)], 28.5.2009, C 119/2-10. https://eur-lex.europa.eu/legal-content/ES/TXT/?uri=celex\%3A5 2009XG0528\%2801\%29.

Gardner, H. (1983). Frames of mind: The theory of multiple intelligences. New York: Basic Books.

Gardner, H. (1985). The mind's new science: a history of the cognitive revolution. New York: Basic Books.

Gardner, H. (1995). Inteligencias Múltiples: La teoría en la práctica. [Multiple Intelligences: Theory in practice]. Barcelona: Paidós Educación.

Gardner, H. (2000). Intelligence reframed: Multiple intelligences for the 21st century. London: Hachette UK.

Gotlieb, R.; Hyde, E., Immordino-Yang, M. H. \& Kaufman, S. B. (2016). Cultivating the socialemotional imagination in gifted education: insights from educational neuroscience. Annals of the New York Academy of Sciences, 1377, 22-31. https://doi.org/10.1111/ nyas. 13165.

Kaufman, S. B.; Reynolds, M. R.; Liu, X.; Kaufman, A. S. y McGrew, K. S. (2012). Are cognitive $g$ and academic achievement $g$ one and the same $g$ ? An exploration on the Woodcock-Johnson and Kaufman test. Intelligence, 40, 123-138. https://doi.org/10.1016/j. intell.2012.01.009.

Martín, C. (1992). Análisis del modelo de inteligencia de Robert J. Sternberg. [Analysis of the intelligence model of Robert J. Sternberg]. Tabanque: Revista Pedagógica, (8), 21-38. https://dialnet.unirioja.es/servlet/articulo?codigo=2254588.

Martínez, A. R.; Castejón, J. L. y Sánchez, M. D. P. (1997). Modelos y estrategias de identificación del superdotado. [Models and estrategies for gifted identification]. En Identificación, evaluación y atención a la diversidad del superdotado (pp. 17-40). Archidona: Ediciones Aljibe.

McClain, M. C. y Pfeiffer, S. (2012). Identification of gifted students in the U. S. today: A look at state definitions, policies, and practices. Journal of Applied School Psychology, 28, 59-88. https://doi.org/10.1080/15377903.2012.643757.

Mönks, F. J. y van Boxtel, H. W. (1988). Los adolescentes superdotados: una perspectiva evolutiva. [Gifted teenagers: an evolutionary perspective]. En J. Freeman (dir.). Los niños superdotados. Aspectos psicológicos y pedagógicos (pp. 306-327). Madrid: Santillana.

Mora, J. A. y Martín, M. L. (2007). La concepción de la inteligencia en los planteamientos de Gardner (1983) y Sternberg (1985) como desarrollos teóricos precursores de la noción 
de inteligencia emocional. [The conception of intelligence in the approaches of Gardner (1983) and Sternberg (1985) as theoretical precursor developments of the notion of emotional intelligence]. Revista de Historia de la Psicología, 28(4), 67-92. https://dialnet. unirioja.es/servlet/articulo?codigo $=2514677$

Olszewski-Kubilius, P., Subotnik, R. F. y Worrell, F. C. (2015a). Antecedent and concurrent psychosocial skills that support high levels of achievement within talent domains. High Ability Studies, 26(2), 195-210. https://doi.org/10.1080/13598139.2015.1095077.

Olszewski-Kubilius, P.; Subotnik, R. F. y Worrell, F. C. (2015b). Repensando las altas capacidades: una aproximación evolutiva. [Rethinking high capacities: an evolutionary approach]. Revista de Educación, 368, 40-65. https://doi.org/10.4438/1988592X-RE-2015-368-293.

Pérez, R. E. (2018). La Medición de la Inteligencia y las Aptitudes en la Identificación del Talento [The Measurement of Intelligence and Skills in Talent Identification]. Anuario de Investigaciones de la Facultad de Psicología, 3(3), 359-368. https://revistas.unc.edu. ar/index.php/aifp/article/view/20741.

Pérez, E. y Medrano, L. A. (2013). Teorías contemporáneas de la inteligencia. Una revisión crítica de la literatura. [Contemporary theories of intelligence. A critical review of the literature]. Psiencia. Revista Latinoaméricana de Ciencia Psicológica, 5(2), 105-118. https://doi.org/10.5872psiencia/5.2.32.

Piaget, J. y Inhelder, B. (1971). Mental Imagery in the Child. London: Routledge \& K. Paul.

Pleiffer, S. (2013). Serving the Gifted: Evidence-Based Clinical and Psychoeducational Practice. New York: Routledge.

Pfeiffer, S. I. (2015). El Modelo Tripartito sobre la alta capacidad y las mejores prácticas en la evaluación de los más capaces: Tripartite Model of Giftedness and Best Practices in Gifted Assessment. [The Tripartite Model on high capacity and best practices in the evaluation of the most capable]. Revista de Educación, 368, 66-95. https://doi. org/10.4438/1988-592X-RE-2015-368-293.

Ramos, J. F. (2008). La respuesta educativa al alumnado con altas capacidades desde el enfoque curricular: del plan de atención a la diversidad a las adaptaciones curriculares individuales. [The educational response to students with high capacities from the curricular approach: from the plan of attention to diversity to individual curricular adaptations]. Faisca, 13(15), 40-49. https://dialnet.unirioja.es/servlet/articulo?codigo=3539199.

Renzulli, J. S. (1978). What makes giftedness? Reexamining a definition. Phi Delta Kappan, 60 (3), 180-184. https://www.researchgate.net/publication/234665343_What_Makes_ Giftedness_A_Reexamination_of_the_Definition.

Renzulli, J. S. (1981). The Revolving-Door Model: A New Way of Identifying the Gifted. Phi Delta Kappan, 62(9), 648-649. https://www.researchgate.net/publication/234596861_ The_Revolving-Door_Model_A_New_Way_of_Identifying_the_Gifted.

Renzulli, J. S. (2012). Reexamining the role of gifted education and talent development for the 21st century: A four-part theoretical approach. Gifted Child Quarterly, 56(3), 150159. https://doi.org/10.1177/0016986212444901.

Reyero, M. y Tourón, J. (2000). En torno al concepto de superdotación: evolución de un paradigma. [Around the concept of giftedness: evolution of a paradigm]. Revista Española de Pedagogía, 215, 7-38. https://dialnet.unirioja.es/servlet/articulo?codigo=23655.

Sahuquillo, P.; Ramos, G.; Pérez, A. y Camino, A. I. (2016). Las competencias parentales en el ámbito de la identificación/evaluación de las altas capacidades. [Parental competencies in the field of identification/evaluation of high capacities]. Profesorado. Revista de 
ALBERTO QUÍLEZ ROBRES Y RAQUEL LOZANO BLASCO

MODELOS DE INTELIGENCIA Y ALTAS CAPACIDADES: UNA REVISIÓN DESCRIPTIVA Y COMPARATIVA

Currículum y Formación del Profesorado, 20(1), 200-217. https://recyt.fecyt.es/index. $\mathrm{php} / \mathrm{profesorado/article/view/52099.}$

Sánchez, L. P.; Cobeñas, E. T. L.; Del Valle Chauvet, L. y Belinchón, E. R. (2008). Más allá del currículum: programas de enriquecimiento extraescolar: La experiencia del programa estrella. [Beyond the curriculum: after-school enrichment programs: The experience of the star program]. Faisca: Revista de Altas Capacidades, 13(15), 4-29. https://dialnet. unirioja.es/servlet/articulo?codigo $=3537454$.

Sosniak, L. A. (1985). A long-term commitment to learning. En B. J. Bloom (Ed.), Developing talent in young people (pp. 477-506). New York: Ballantine.

Sternberg, R. J. (1985). Beyond IQ: A triarchic theory of human intelligence. Cambridge: Cambridge University Presss.

Sternberg, R. J. (1999). Intelligence as developing expertise. Contemporary Educational Psychology, 24, 359-375. https://doi.org/10.1006/ceps.1998.0998.

Sternberg, R. J. y Davidson, J. E. (Eds.) (1986). Conceptions of giftedness. New York: Cambridge University Press.

Sternberg, R. J.; Grigorenko, E.; Ferrando, M.; Hernández, D.; Ferrándiz, C. y Bermejo, R. (2010). Enseñanza de la inteligencia exitosa para alumnos superdotados y talentos. [Successful intelligence teaching for gifted students and talents]. Revista Electrónica Interuniversitaria de Formación del Profesorado, 13(1), 111-118. https://dialnet.unirioja.es/servlet/articulo?codigo $=3163513$.

Sternberg, R. J.; Kaufman, J. C. y Grigorenko, E. L. (2008). Applied intelligence. Cambridge: University Press.

Subotnik, R. F.; Olszewski-Kubilius, P. y Worrell, F. C. (2011). Rethinking giftedness and gifted education: A proposed direction forward based on psychological science. Psychological science in the public interest, 12(1), 3-54. https://doi.org/10.1177/1529100611418056.

Subotnik, R. F.; Olszewski-Kubilius, P. y Worrell, F. C. (2012). A proposed direction forward for gifted education based on psychological science. Gifted Child Quarterly, 56(4), 176188. https://doi.org/10.1177/0016986212456079.

Tannenbaum, A. J. (1986). Giftedness: A psychosocial approach. En R. Sternberg, y J. Davidson, J. (Eds.). Conceptions of giftedness (pp. 21-52). Cambridge University Press.

Tannenbaum, A. J. (2003). Nature and nurtures of giftedness. En N. Colángelo y G. A. Davis (Eds.). Handbook of Gifted Education [Manual de educación para dotados] (3nd ed.) (pp. 45-59). Boston: Pearson Education.

Triglia, A.; Regader, B. y García-Allen, J. (2018). ¿Qué es la inteligencia? Del CI a las inteligencias múltiples. [What is intelligence? From ci to multiple intelligences]. Barcelona: EMSE Publishing.

Van Boxtel, H. W. y Mönks, F. J. (1992). General, social, and academic self-concepts of gifted adolescents. Journal of youth and adolescence, 21(2), 169-186. https://doi.org/10.1007/ BF01537335.

Villamizar, G. y Donoso, R. (2013). Definiciones y teorías sobre inteligencia. Revisión histórica. [Definitions and theories about intelligence. Historical review]. Psicogente, 16(30), 407-423. https://dialnet.unirioja.es/servlet/articulo?codigo=6113904. 
